\title{
Signal Simulation on Static Test of Guidance Station of One Antiaircraft Missile
}

\author{
Ke LI, Lian Yan SHI, Xin HE, Jin LUO \\ 4th department, Shijiazhuang Mechanic Engineering College, Shijiazhuang, HEBEI, CHINA, 050003
}

\begin{abstract}
In order to improve trainees' operational quality of static test of guidance station of one antiaircraft missile, a static testing simulator is necessary to gain the goal. It is obvious that the key problem of developing the simulator is how to generate the static testing signals of the simulator. Aiming to solve the problem, at first, the paper adopts a standard called signal testing definition(STD), which is applied to describe simulating signals used for static testing simulation of guidance station of one antiaircraft missile; then those simulating signals for the static testing simulation are implemented based on the platform Labwindows/CVI, and D/A converters are employed for generating physical static testing signals, which are outputted through operational panels or interfaces of the static testing simulator. So the mentioned process could be helpful to realize the static testing simulator of guidance station of one antiaircraft missile.
\end{abstract}

\section{Introduction}

People said the more training, the high operational quality. In order to improve trainees' operational quality of static test of the guidance station of one antiaircraft missile, a static testing simulator is necessary to gain the goal. It is obvious that the key problem of developing the simulator is how to generate the static testing signals of the simulator. The paper tries to solve the key problem to help develop the simulator.

\section{Simulating requirements analysis}

The static test of the guidance station of one antiaircraft missile has a number of 320 plus test project, it is one of the equipment daily maintenance project in order to test whether working state of the equipment is normal, The method is to apply incentives in different testing points of the equipment, then to measure corresponding outputs to judge state of the equipment. Therefore, the realization of the static testing simulator or test simulating function is to produce the various all kinds of response signals of the equipment. According to the characteristics of the static testing training and requirements of the guidance station of one antiaircraft missile, analysis on simulation requirements would be on the following three facets for developing the static testing simulator of one antiaircraft missile.

\subsection{Classification of simulating signals}

(1) Transient voltages and currents: the initial voltage and current for the equipment;
(2) The DC voltages and currents: the stable voltage and current for the equipment;

(3) AC voltages: output information, noise and other signals;

(4) Controlled voltages: the equipment controlled voltage;

(5) Resistance parameters: circuit's resistance of the equipment, breakover resistance, etc.

(6) Complex waveform signals: the rectangular wave with adjustable width or frequency modulation etc. controlled by the incentive voltage or power time, as well as various instruction outputting, signals such as pulse front and back edge etc.;

(7) Sound and light signals: sound and light output when the equipment works;

(8) The state signals: they are controlled by frequency, voltage, time, or other inventive signals, in other words, they are changing signals of controlled state or time;

(9) Visual signals: focus changing, target, capture line, self-check lamp, etc.

(10) Other signals: output signals of the signal generator without power, self-check signal of voltage stabilizer circuit, etc.

\subsection{Analysis on signal relationship}

The static testing operation of the equipment is not only to achieve the simulation of various signals or parameters, but also to simulate logical function of the actual system, signal control relationship, etc., and analysis and identification on various incentives.

(1) Relationship among the launching control program and the control instructions; 
(2) Relationship among information signals, serial data and instructions;

(3) Visual signals and detecting states;

(4) Relationship between serial commands and instructions;

(5) Control relationship among other signals.

\subsection{Signal stability}

(1) AC voltage: $2.5 \%$ of the nominal value;

(2) DC voltage: $2 \%$ of the nominal value;

(3) DC current: $1.5 \%$ of the nominal value;

(4) Frequency/time: $0.1 \%$ of the nominal value;

(5) Insulating resistance: $1 \%$ of the nominal value;

(6) Coefficient of instructions: $1 \%$ of the nominal value.

\section{3 signal description and modeling}

One signal of static test of the guidance station of one antiaircraft missile is selected as an example, with which the method of signal modeling and generating in the paper is depicted in detail. The selected signal waveform is shown as Figure 1.

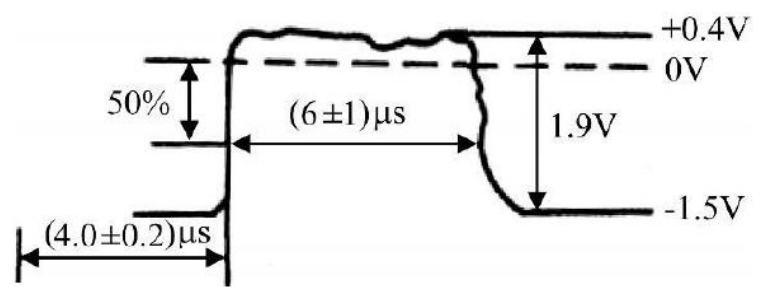

Figure 1. Selected irregular waveform

\subsection{Signal simulating model based on signal testing definition [1]}

The characteristics of signal testing definition (STD, IEEE1641) include:(1)it provides unique definition for all kinds of signals;(2)it provides mathematical basis for defining various signals, which would facilitate signal accurate output, recreating and usage in other testing system;(3)it provides a structure for signal combination to construct the complex signals;(4)it supports to build the scalable signal set library for more than one similar application field;(5)it offers a method of signal definition, usage and control, which could be applied by a few various programming languages;(6)the test program in accordance with STD could be applied to any test system with the same STD signal.

STD signals defined structure is shown as figure 2 . Signal test and definition of STD standard are divided into signal modeling language (SML), basic signal components (BSC), test signal framework (TSF) and test procedure language (TPL). BSC layer provides the basic structure modules to build the complex signals, but its basic class components are simple signals, and unit under test (UUT) measurement signals are often more complex than what one basic class component of BSC could represent. So STD standard provides a mechanism to construct complex signals with several basic class components of BSC.

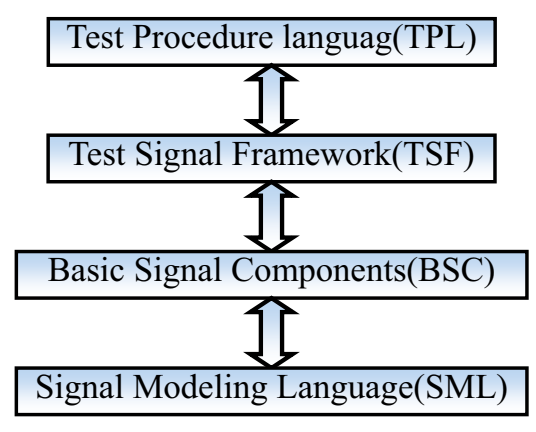

Figure 2. STD layered structure

\subsection{Construction of signal model}

The model structure of a selected irregular signal (its waveform is shown as fig.1), which is constructed based on a basic class component of BSC, is shown as Figure 3.

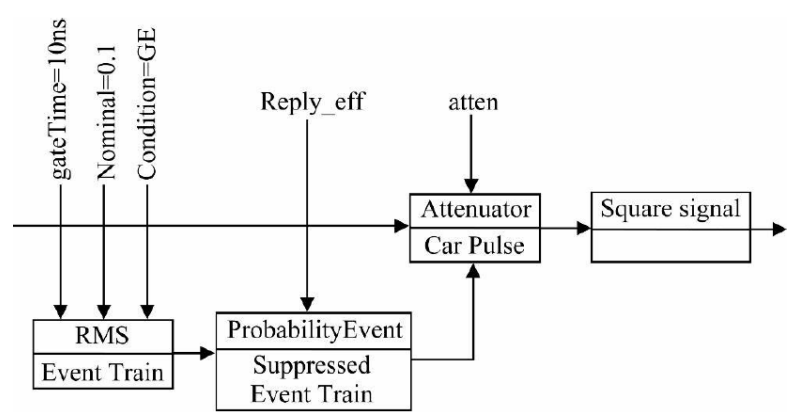

Figure 3. Block diagram of an irregular signal based on BSC of STD standard

Each module of the signal structure (RMS, probability event, attenuator, Signal delay) is one of BSC components. The small amplitude sinusoidal signal is the input signal; RMS calculates whether the root mean square value of the transmitted signal is greater than $0.1 \mathrm{~V}$ in an assumed $10 \mathrm{~ns}$ time, an output event would be generated while the root mean square value of the transmitted signal is over $0.1 \mathrm{~V}$. The reply_eff (effective rate of a response) of probability event component determines the response efficiency of one event by a value selected from 0 to $100 \%$, then one selective generated event that produces a random disturbance signal would be sent to one gate of the attenuator; When the transmitted signal is attenuated by "atten" times, its amplitude value would be in the required range, the signal is loaded on the square wave signal, and the output is the required signal waveform.

\section{Simulating signal generation of static test}

The platform LabWindows/CVI is adopted to generate simulating signals of static test, which are described by XLM $[2,3]$. 


\subsection{LabWindows/CVI code}

The main () function is the entrance to the program; its function is to initialize and display program, to load user panels. The codes are as follows:

SineWave ( 1000, 4, 30.8125e-3, \&phase, sin1 ); SineWave ( 1000, 0. 5, 50.8125e-3, \&phase, $\sin 2$ ); WhiteNoise ( 1000, 0. 05, 100, noise1 );

WhiteNoise ( $1000,0.15,100$, noise 2$)$;

$\operatorname{Ramp}(6,0.4,-0.95$, xiepo1 );

fo $r(i=0 ; i<8000 ; i++)$

data $[\mathrm{i}]=0$;

fo $r(\mathrm{i}=0 ; \mathrm{i}<38 ; \mathrm{i}++)$

data $[\mathrm{i}]=-1.5$;

for $(\mathrm{i}=38 ; \mathrm{i}<41 ; \mathrm{i}++)$ data $[\mathrm{i}]=\sin 1[\mathrm{i}-14]+2.5$;

for $(i=41 ; i<45 ; i++)$ data $[i]=\sin 1[i-3]-3.6$; for $(i=45$;

$\mathrm{i}<107 ; \mathrm{i}++)$ data $[\mathrm{i}]=0.4+$ noise $1[\mathrm{i}]$;

for $(\mathrm{i}=107 ; \mathrm{i}<113 ; \mathrm{i}++$ )

$\operatorname{data}[\mathrm{i}]=\operatorname{xiepo} 1[\mathrm{i}-107]+$ noise $1[\mathrm{i}]$;

for $(\mathrm{i}=113 ; \mathrm{i}<119 ; \mathrm{i}++)$ data[i] $=\sin 2[\mathrm{i}-1]-0.95$; for $(\mathrm{i}=119 ; \mathrm{i}<151 ; \mathrm{i}++)$ data[i] $=-1.5$;

SetCtrlAttribute( pane,1 PANEL_GRAPH,

ATTR_XAX IS_GA IN, 1.0/10000);

SetCtrlAttribute (pane,1 PANEL_GRAPH, ATTR_XPREC ISION, 5 ); PlotY( pane Hl and le, PANEL_GRAPH, data, 150, VAL_DOUBLE, VAL _THIN_LINE, VAL_EM ${ }^{-}$PTY_SQUAR E, VAL_SOL ID, 1, VAL_RED );

The result of the program to be run is shown as Figure 4. Based on the principle introduced as section 2.2, a random small amplitude sinusoidal signal is loaded on a basic square wave to form small amplitude waveforms, and carries on the other processing technology, as shown in figure 4 wave charts, as can be seen from the diagram, using LabWindows/CVI waveform to get the waveform is similar to the original waveform (figure 1).

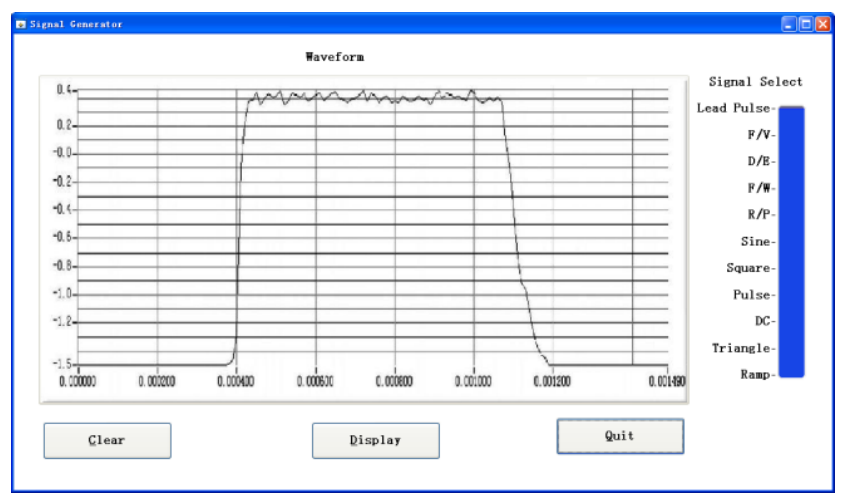

Figure 4. Irregular waveform

\subsection{Simulating signal output of static test}

In the oscilloscope based on the LabWindows/CVI, the waveform file generated in terms of above method is loaded to drive the oscilloscope to produce physical waveform $[4,5]$.

\section{Conclusions}

Aiming at implement of a static test training simulator of one antiaircraft missile, the paper firstly commences the system requirement analysis; secondly, the simulated waveforms of the simulator are depicted and modeled with XML language, specifically all of signal simulation models are established on STD standard. At last, the physical signals are outputted with LabWindows/CVI and $\mathrm{D} / \mathrm{A}$. The relevant experiments proved the idea of the paper is feasible, and conclusions of the paper could help to realize a static test training simulator of one antiaircraft missile.

\section{References}

1. Yang Sen, Meng Chen, Wang. ChengResearch and Application of ATS Test Description Language Based on STD Standard. Computer Measurement \& Control, 2010, 3(25).

2. LIU Junhua. VISUAL INSTRUMENT PROGRAM LabWindows/CVI COURSE [M]. BEIJING: ELECTRONIC INDUSTORY PRESS, 2001.

3. QIAN Neng. $\mathrm{C}++{ }^{\text {t }}$ PROGRAM DESIGN COURSE [M]. BEIJIN:TSINGHAU PRESS, 2001.

4. YAN Bo. Research and Development of the General LFM Signals Generating System Based on DDS Technology [D]. CHENGDU: UNIVERSITY of ELECTRONIC SCIENCE AND TECHNOLOGY OF CHINA

5. TENG $\mathrm{Xu}, \mathrm{HU}$ Zhiang. COUNTERMEASURE PRACTICAL TECHNOLOGY OF ELECTRIC SYSTEM [M]. BEIJIN: NATIONAL DEFENCE INDUSTORY PRESS, 2004. 